\title{
Appearance of Light Clusters in Post-bounce Evolution of Core-Collapse Supernovae
}

\author{
Kohsuke Sumiyoshi \\ Numazu College of Technology, Ooka 3600, Numazu, Shizuoka 410-8501, Japan \\ Gerd Röpke \\ University of Rostock, Institut für Physik, Universitätsplatz 1, 18051 Rostock, Germany
}

(Dated: November 1, 2018)

\begin{abstract}
We explore the abundance of light clusters in core-collapse supernovae at post-bounce stage in a quantum statistical approach. Adopting the profile of a supernova core from detailed numerical simulations, we study the distribution of light bound clusters up to alpha particles $(2 \leq A \leq 4)$ as well as heavy nuclei $(A>4)$ in dense matter at finite temperature. Within the frame of a clustermean field approach, the abundances of light clusters are evaluated accounting for self-energy, Pauli blocking and effects of continuum correlations. We find that deuterons and tritons, in addition to ${ }^{3} \mathrm{He}$ and ${ }^{4} \mathrm{He}$, appear abundantly in a wide region from the surface of the proto-neutron star to the position of the shock wave. The appearance of light clusters may modify the neutrino emission in the cooling region and the neutrino absorption in the heating region, and, thereby, influence the supernova mechanism.

PACS numbers: 21.45.-v,21.65.-f,26.50.+x,97.60.Bw
\end{abstract}

The formation of clusters in nuclear matter is one of pivotal phenomena in nuclear structure and heavy ion collisions. Light clusters such as ${ }^{4} \mathrm{He}$ appear also in astrophysical environments at certain densities and temperatures. If they exist abundantly, they may change the properties of dense matter and contribute to new reactions controling the dynamical evolution of stars.

In the explosion mechanism for core-collapse supernovae, which has been a long standing problem despite continuous research for decades [1], the existence of ${ }^{4} \mathrm{He}$ and nuclei in the heating region has been proposed to be a possible agent to help the stalled shock wave via the neutrino heating mechanism [2, 3]. In most of the recent numerical simulations of core-collapse supernovae studying the gravitational collapse of massive stars, the shock wave produced by a core bounce stalls on the way and needs some mechanism to revive the outward propagation of the shock wave. The heating source through neutrino reactions in dense matter behind the stalled shock is one of key issues in sophisticated numerical simulations [4, 5]. It has been shown that neutrino- ${ }^{4} \mathrm{He}$ reactions may marginally affect the standing accretion shock instability and consequently the supernova shock revival [6].

Besides ${ }^{4} \mathrm{He}$, the light clusters such as deuterons, tritons and ${ }^{3} \mathrm{He}$ have not been included in numerical simulations of supernovae so far. Those light clusters may become new agents for absorption and emission of neutrinos, which can carry energy away and heat the material in the important phase of supernova dynamics. In the currently available sets of equations of state (EOS) for supernova simulations such as the Shen-EOS 7] and the Lattimer-Swesty EOS [8], dense matter is described as a mixture of neutrons, protons, ${ }^{4} \mathrm{He}$, and one representative species of nuclei. Since the occurrence of light clusters in addition to ${ }^{4} \mathrm{He}$ is a natural outcome in nuclear statistical equilibrium, one should clarify whether they are also present in realistic profiles of a supernova core.
A consistent description of the composition of hot and dense nuclear matter is preferable for the rigorous treatment of neutrino transport in the post-bounce evolution of core-collapse supernovae. Progress in the description of clusters in dense matter [9, 10] based on a generalized Beth-Uhlenbeck approach [11, 12] enables us to evaluate the abundance of deuterons, tritons, and helium nuclei altogether within a microscopic approach. The mixture of tritons and ${ }^{3} \mathrm{He}$ and their neutrino reactions have recently been studied under the condition of fixed temperatures and proton fractions [13], but without deuterons and heavy nuclei.

In this paper, we report the appearance of light clusters in dense matter of supernova cores adopting the microscopic treatment of clusters developed within a quantum statistical approach. We aim at the unification of the quasiparticle approach such as the relativistic mean-field theory [7, 14], which may be adequate at high baryon densities, and the cluster approach including the virial expansion 11, 12, 13, 15, 16] which may be applicable in the low-density limit.

The EOS of nuclear matter $n_{i}\left(T, \mu_{p}, \mu_{n}\right)$ giving the total particle densities of protons and neutrons, $i=p, n$, respectively, as function of temperature $T$ and chemical potentials $\mu_{i}$, is obtained from the spectral function $A(1, \omega)$, with $1=\left\{\vec{k}_{1}, \sigma_{1}, \tau_{1}\right\}$ denoting momentum, spin and isospin of a single nucleon. In the low-density, lowtemperature region, a nonrelativistic Hamiltonian approach is possible which is based on a nucleon-nucleon interaction $V\left(12,1^{\prime} 2^{\prime}\right)$. Well known examples are the Bonn and Paris potentials or its separable representations, reproducing empirical properties such as scattering phase shifts. Within this quantum statistical approach, the baryon density is calculated as

$$
n_{B}\left(T, \mu_{p}, \mu_{n}\right)=\sum_{1} \int \frac{d \omega}{2 \pi} \frac{1}{\mathrm{e}^{\left(\omega-\mu_{1}\right) / T}+1} A(1, \omega) .
$$


Further thermodynamic quantities such as pressure $p\left(T, \mu_{p}, \mu_{n}\right)$ or free energy density $f\left(T, n_{p}, n_{n}\right)$, being potentials, are obtained by integrations.

The spectral function is related to the self-energy $\Sigma$,

$$
A(1, \omega)=\frac{2 \operatorname{Im} \Sigma(1, \omega-i 0)}{\left[\omega-p_{1}^{2} / 2 m_{1}-\operatorname{Re} \Sigma(1, \omega)\right]^{2}+[\operatorname{Im} \Sigma(1, \omega-i 0)]^{2}} .
$$

Besides the quasiparticle contribution at $E^{\mathrm{qu}}(1)=$ $p_{1}^{2} / 2 m_{1}+\operatorname{Re} \Sigma\left(1, E^{\mathrm{qu}}(1)\right)$ it contains also the contribution of bound states and continuum correlations included in $\operatorname{Im} \Sigma(1, \omega-i 0)$, so that within a generalized BethUhlenbeck approach a virial expansion can be performed in the low-density region. After the cluster decomposition of the self-energy [11, 15], the contributions of the different clusters are treated in a systematic way.

The cluster decomposition for the self-energy involves the calculation of the $A$-nucleon T matrix which obeys a corresponding Bethe-Salpeter equation. Two-particle correlations in nuclear matter can be accounted for by considering the two-particle Green function in ladder approximation. The corresponding Bethe-Salpeter equation contains self-energy and Pauli-blocking terms [12]. The cluster-mean field approximation [15] yields a wave equation which describes also arbitrary clusters in a clustered medium. For the two-particle system, neglecting correlations in the surroundings, the following wave equation results

$$
\begin{aligned}
& {\left[E^{\mathrm{qu}}(1)+E^{\mathrm{qu}}(2)-E_{2, \lambda}^{\mathrm{qu}}(P)\right] \psi_{\lambda, P}(12)} \\
& +[1-f(1)-f(2)] \sum_{1^{\prime} 2^{\prime}} V\left(12,1^{\prime} 2^{\prime}\right) \psi_{\lambda, P}\left(1^{\prime} 2^{\prime}\right)=0 .
\end{aligned}
$$

The medium Fermi distribution function $f(1)=$ $\left\{\exp \left[E^{\mathrm{qu}}(1) / T-\hat{\mu}_{1} / T\right]+1\right\}^{-1}$ contains the effective chemical potential $\hat{\mu}_{1}$ which is determined by the total proton or neutron density calculated in quasiparticle approximation, $n_{i}=\sum_{1} f(1) \delta_{\tau_{1}, i}$. It describes the occupation of the phase space neglecting any correlations in the medium. For the parameter values $T, n_{B}$ considered below the account of correlations in the medium gives no significant changes in the spectral function [15].

The quasiparticle energies $E^{\mathrm{qu}}(1)$ are self-consistently calculated if we evaluate the self-energy or the corresponding $\mathrm{T}$ matrix. Using the cluster decomposition and restricting only to the two-particle contribution [12], the results coincide with the Brueckner K matrix including hole-hole contributions. The quasiparticle energies can be parametrized and directly related to empirical data on finite nuclei. An appropriate parametrization can be found on the basis of relativistic mean-field theories [7, 14] which also involves the results of relativistic Brueckner-Hartree Fock calculations [17]. We will use the TM1 parametrization here.

From the solution of this in-medium two-particle Schrödinger equation (2) the scattering states and possibly the bound states are obtained. Due to the self-energy shifts and the Pauli blocking, the binding energy of the deuteron $E_{d}\left(P ; T, \mu_{p}, \mu_{n}\right)$ as well as the scattering phase shifts $\delta_{\lambda}\left(E, P ; T, \mu_{p}, \mu_{n}\right)$ in the respective isospin singlet or triplet channel $\lambda$, will depend on the temperature and the chemical potentials [12, 18].

In-medium Schrödinger equations similar to Eq. (2) containing quasiparticle shifts and Pauli blocking terms are derived for higher clusters with mass number $A$ and charge $Z[15]$. The shift of the bound state energies $E_{A, Z}^{\mathrm{qu}}\left(P ; T, n_{i}\right)=E_{A, Z}+\Delta_{A, Z}^{\mathrm{SE}}+\Delta_{A, Z}^{\mathrm{Pauli}}+\Delta_{A, Z}^{\mathrm{Coul}}$, containing in addition to the single-particle self-energy shift $\Delta_{A, Z}^{\mathrm{SE}}$ and the Pauli blocking term $\Delta_{A, Z}^{\text {Pauli }}$ also the Coulomb shift $\Delta_{A, Z}^{\text {Coul }}$, can be calculated within perturbation theory. Besides the quasiparticle shift at zero momentum $\Delta_{A, Z}^{\mathrm{SE}, 0}=Z E_{p}^{\mathrm{qu}}(0)+(A-Z) E_{n}^{\mathrm{qu}}(0)$ which can be included into the chemical potential, there is the contribution due to the effective mass $m^{*}$. Assuming for light clusters $2 \leq A \leq 4$ a Gaussian wave function with the nucleonic rms radii $\left\langle r^{2}\right\rangle_{A, Z}$, the self-energy shift results as $\Delta_{A, Z}^{\mathrm{SE}}=\Delta_{A, Z}^{\mathrm{SE}, 0}+3(A-1) \hbar^{2} b_{A, Z}^{2}\left(m-m^{*}\right) /\left(8 m^{2}\right)$ after introducing Jacobian coordinates and separating the c.o.m. motion, where $b_{A, Z}^{2}=3(A-1) /\left(A\left\langle r^{2}\right\rangle_{A, Z}\right)$. With Gaussian wave functions we obtain the following estimate for the Pauli blocking shift

$$
\Delta_{A, Z}^{\text {Pauli }}=\int \frac{q^{2} d q \mathrm{e}^{-\frac{2 A q^{2}}{b^{2}(A-1)}}\left[|E|+\frac{\hbar^{2}}{2 m}\left(\frac{A}{A-1} q^{2}+\frac{3(A-2)}{4} b^{2}\right)\right]}{\left(\mathrm{e}^{\left[\frac{\hbar^{2}}{2 m}\left(\frac{P}{A}+q\right)^{2}-\hat{\mu}_{i}\right] / T}+1\right)\left[\frac{A-1}{A}\right]^{3 / 2} \frac{\sqrt{\pi}}{8} b^{3}} .
$$

Similar expressions [19] can be given for the weakly bound clusters with $5 \leq A \leq 11$. The Coulomb shift

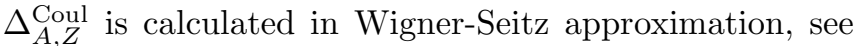
also [8, 19]. Within the parameter values considered below, the influence of the Coulomb corrections on the composition is small.

For heavier clusters with mass numbers $A \geq 12$, the self-energy and Pauli-blocking shifts become less important and will be neglected here. The heavier clusters repel the nuclear matter so that the mean-field effects are mostly produced by the other nucleons within the cluster and are contained in the cluster binding energy. For a more detailed consideration see [19].

Using the cluster decomposition of the self-energy, from Eq. (11) a generalized Beth-Uhlenbeck formula can be derived which is formally similar to the virial expansion of the EOS

$$
n_{B}\left(T, \mu_{p}, \mu_{n}\right)=\sum_{A=1, Z}^{\infty} A n_{A, Z}\left(T, \mu_{p}, \mu_{n}\right) .
$$

Introducing the Fermi or Bose distribution function $f_{A, Z}(E)=\left[\mathrm{e}^{\left(E-Z \mu_{p}-(A-Z) \mu_{n}\right) / T}-(-1)^{A}\right]^{-1}$, the EOS (41) contains the single-particle contribution $n_{1, Z}=2 \sum_{P} f_{1, Z}\left(E^{\mathrm{qu}}(P)\right)$, the two-particle contributions $n_{2, Z}=n_{2, Z}^{\text {bound }}+n_{2, Z}^{\text {scat }}$ (consisting of the contribution of medium-modified deuterons and scattering states in the isospin singlet and triplet channel, see [12, 18]), as well as the contribution of all higher clusters. 
The bound state contribution of the $A$-nucleon cluster to the EOS (4) has the form [12, 16, 19] $n_{A, Z}=$ $\sum_{P, \lambda}\left[f_{A, Z}\left(E_{A, Z, \lambda}^{\mathrm{qu}}(P)\right)-f_{A, Z}\left(E_{A, Z}^{\mathrm{cont}}(P)\right)\right]$ where $\lambda$ denotes the internal degrees of freedom such as spin and excitation states. The summation over the c.o.m. momentum $P$ runs only over the region where the in-medium binding energy $E_{A, Z, \lambda}^{\mathrm{qu}}(P)$ is lower than the edge of the continuum $E_{A, Z}^{\mathrm{cont}}(P)=A E^{\mathrm{qu}}(P / A)$. The summation over all excited states of a large cluster can be replaced by an intergal over the excitation energy using the density of states, see [8, 19].

Of importance is the contribution of continuum correlations which can be represented by the scattering phase shifts. Extended investigations have been performed with respect to the contribution of two-nucleon phase shifts [12]. They reduce the contribution of the bound states. Partial integration, using the Levinson theorem, gives the second contribution $f_{A, Z}\left(E_{A, Z}^{\text {cont }}(P)\right)$. Thus, there is no jump in the EOS when a bound state merges with the continuum if, e.g., the baryon density is increased. The remaining correlations in the continuum are compensated to a large extent by the contribution of interaction to the quasiparticle shift and will be neglected. Explicitly this can be shown for the contribution of the two-nucleon singlet and triplet scattering phase shift contributions.

In order to explore the fraction of nucleons bound in light clusters, we utilize a snapshot of a supernova core at post bounce stage, which is important for setting the revival condition of a shock wave and the resulting explosion. We use the profile obtained by the detailed numerical simulation [20] of the gravitational collapse and core bounce for a $15 \mathrm{M}_{\odot}$ pre-supernova star adopting the Shen EOS [7].

Figure 1 displays the profiles of density $(\rho)$, electron fraction $\left(Y_{e}\right)$ and temperature $(T)$ at $150 \mathrm{~ms}$ after the core bounce. The shock wave is stalled at $\sim 130 \mathrm{~km}$. The proto-neutron star is just born at the center and matter is falling down onto it through the shock wave. The neutrino sphere, where the neutrinos are emitted, is located at $20-80 \mathrm{~km}$ depending on energies and species. The electron fraction is $\sim 0.5$ in the iron nuclei region at $r \geq 100 \mathrm{~km}$ and becomes smaller (neutron rich) for dissociated nucleons inside due to the balance under beta equilibrium including neutrinos. Note that the electron fraction is the same as the total proton fraction due to charge neutrality.

At the shock wave, the iron nuclei are dissociated into ${ }^{4} \mathrm{He}$, and, subsequently, nucleons at high temperatures due to the shock heating. We remark that the nuclear statistical equilibrium is maintained during the dynamics because the temperature and density are high at the region of current interest and, therefore, the compositional change proceeds fast enough via charged particle reactions. During this process of dissociation, light clusters may appear in addition to ${ }^{4} \mathrm{He}$ and can modify the cooling and heating rates through weak reactions with leptons. Conventionally, only nucleons are considered for

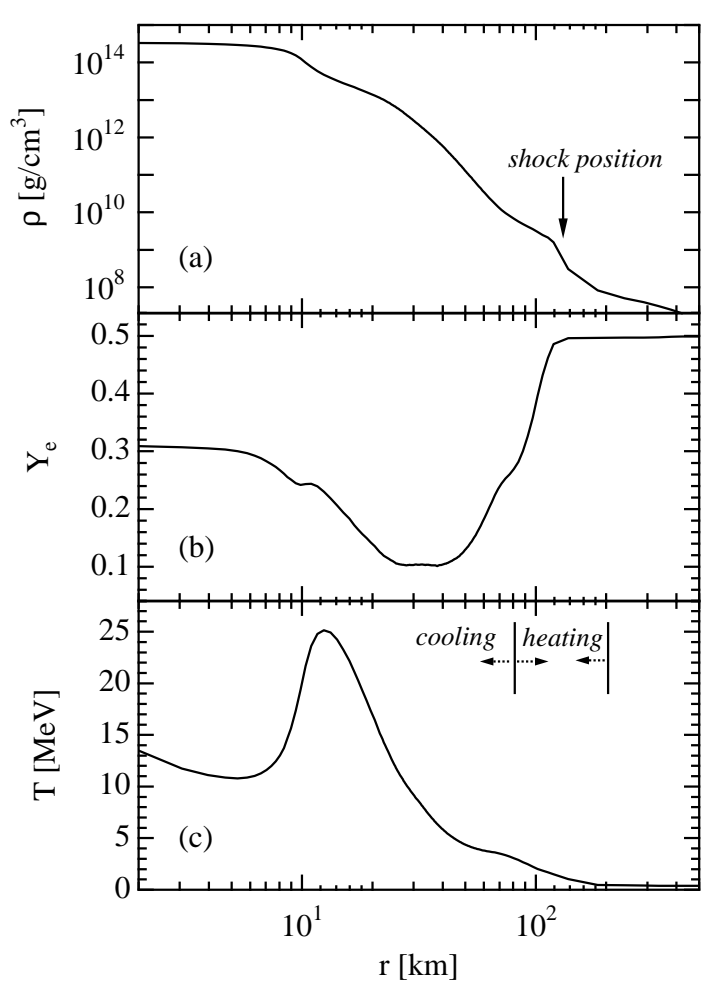

FIG. 1: Density (a), electron fraction (b) and temperature (c) profile of the supernova core at $150 \mathrm{~ms}$ after core bounce as a function of the radius. The position of shock wave and the boundaries of heating and cooling regions are indicated in the top and bottom panels.

the cooling process via neutrino emission at the surface of proto-neutron star (cooling region) and the heating process via neutrino absorption behind the shock wave (heating region).

We solved the EOS (4) for given profiles of $T, \rho, Y_{e}$ as shown in figure 1. The masses of nuclei are taken from Audi and Wapstra 21]. The chemical potentials $\mu_{p}, \mu_{n}$ are determined in a self-consistent way. The abundances of the different clusters have been calculated, and fractions $X_{A, Z}=A n_{A, Z} / n_{B}$ of nucleons bound in deuterons, tritons, ${ }^{3} \mathrm{He},{ }^{4} \mathrm{He}$ as well as the fraction $X_{A}$ of nucleons bound in nuclei with $A \geq 5$ are shown in figure 2 .

The contributions of free protons and free neutrons to the baryon density follows nearly the behavior given by Shen et al. 7, 20]. In the inner region $(r<30 \mathrm{~km})$ where the density and temperature become high, dominant clusters are deuterons and tritons because of the high neutron fraction. Here the main contribution to $X_{A}$ is from $A=5$. In the outer region $(r>100 \mathrm{~km})$, ${ }^{4} \mathrm{He}$ and heavy nuclei become abundant. In the region between, the deuteron fraction is relatively high and the fraction of ${ }^{4} \mathrm{He}$ rises to about $90 \%$ at $r \sim 150 \mathrm{~km}$, before it goes down when the heavier clusters, $A \geq 5$, are formed. The wiggle of $X_{A}$ near $r \sim 150 \mathrm{~km}$ is caused by the weak binding energy of nuclei with $5 \leq A \leq 11$ 


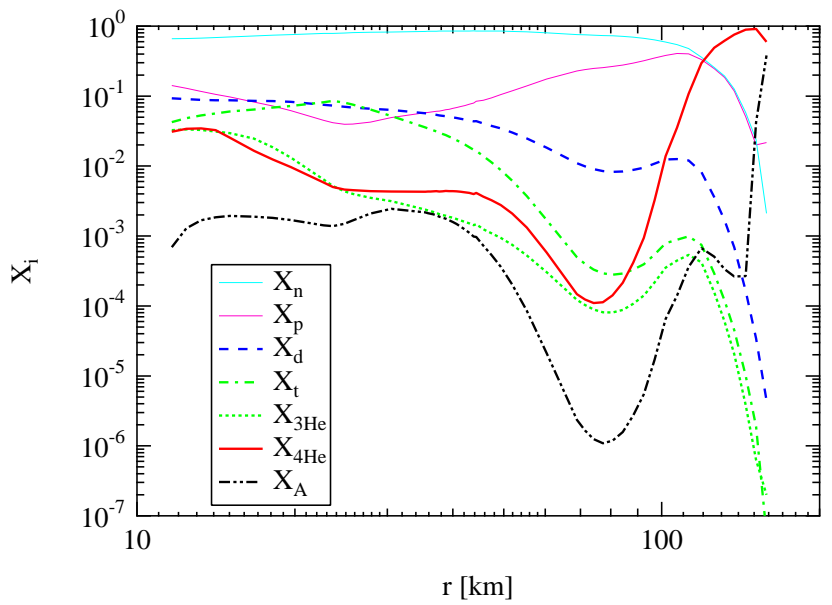

FIG. 2: (Color online) Mass fraction $X_{i}$ of light clusters as function of the radius for the post-bounce supernova core shown in Fig. 1.

which are of relevance there. At larger radii, the strongly bound nuclei of the iron region will dominate.

The appearance of light clusters in supernova cores at post-bounce stage indicates that there are additional weak reactions involving them which will have influence on the supernova dynamics. The neutrino interactions on light nuclei, which appear inside the proto-neutron star at $\sim 10 \mathrm{~km}$, will contribute to the opacity. This may lead to a modification of the diffusion coefficient for neutrinos, the time scale of neutrino diffusion and the location of neutrino sphere, where most of the neutrinos start streaming freely. The light nuclei at $\sim 60$ $\mathrm{km}$ in the cooling region are possible sources of neutrino emission through electron and positron captures. They will modify the neutrino luminosities and spectra because the emission rates per nucleon for light nuclei may be effectively smaller than the large emission rates for nucleons. Medium effects on the neutrino emissivity have been considered in [22] using the many-particle approach described above. In fact, the abundance of nucleons is reduced by about $10 \%$ due to the appearance of light clusters and the corresponding neutrino luminosities may be lowered. We expect that these modifications of the neutrino flux and energy change the neutrino heating rates.

The light clusters in the heating region behind the shock wave become the target compositions for neutrino absorption and will modify the heating rate to assist the revival of shock wave. The dominance of ${ }^{4} \mathrm{He}$ at $\sim 150$ $\mathrm{km}$ in the heating region may contribute to the heating rate via neutrino absorption as discussed by Haxton et al. 2, 3]. It is interesting to see that the fraction of ${ }^{4} \mathrm{He}$ in the current approach is larger than the value in the Shen EOS [20] where the low binding energies for the clusters with $5 \leq \mathrm{A} \leq 11$ are not accounted for.
Deuterons become more abundant than ${ }^{4} \mathrm{He}$ at $r<100$ $\mathrm{km}$ and dominate over other nuclear species in the heating region. The neutrino absorption cross section on deuteron is much larger than that for ${ }^{4} \mathrm{He}$ [23, 24, 25], therefore, deuterons may become more effective targets for the heating than ${ }^{4} \mathrm{He}$. Neutrino heating processes on triton and ${ }^{3} \mathrm{He}$ should be studied whether they may contribute as well [13]. When nucleons appear together with light clusters, the heating via the neutrino absorption on nucleon is dominant since the absorption cross section on nucleon is largest among others. In this case, the appearance of light clusters reduces the total heating rate. These processes with light clusters will influence the neutrino-heating mechanism in the revival of the shock wave for a successful explosion.

We have adopted the spherically symmetric snapshot at $150 \mathrm{msec}$ after bounce as an exemplary case. Other time sequences and multi-dimensional aspects will be studied in our subsequent paper to examine whether light clusters appear generally in a significant amount. It is known that nuclei appear more abundantly behind the shock wave in the hydrodynamical instability and the neutrino- ${ }^{4} \mathrm{He}$ reactions may contribute to the heating [6]. It would be interesting to study the fractions of light clusters in profiles with wider ranges of density and temperature in multi-dimensional simulations. When the reaction time scale becomes too long to achieve the equilibrium in fast flows, one needs to treat further the dynamical formation or destruction of light clusters.

Finding the light clusters in supernova cores motivates detailed studies on weak reactions regarding light clusters. Their reactions must be taken into account, in principle, in any neutrino transfer simulation of core-collapse supernovae and proto-neutron star cooling. It would be preferable to construct the supernova EOS table taking into account the formation of light clusters. The approach given here merges a low-density virial expansion with a quasiparticle approach at higher densities. Since the appearance of light clusters is a natural outcome in dense matter, their existence has to be considered in critical studies not only of the supernova mechanism, but also other problems in nuclear physics.

The authors express their sincere gratitude for the hospitality of Max Planck Institut für Astrophysik, where the new idea to write this paper comes out during their research stay. They are grateful especially to W. Hillebrandt and H.-T. Janka for fruitful discussions and local supports. K. S is grateful to K. Kotake, S. Yamada, H. Suzuki, H. Shen and H. Toki for the collaborations on the equation of state and the supernova simulations. G.R. thanks D. Blaschke, J. Natowitz, P. Schuck, S. Shlomo, S. Typel and H. Wolter for discussions. The numerical simulations were performed at NAOJ (iks13a, uks06a), JAEA and YITP. This work is partially supported by the Grants-in-Aid for the Scientific Research (18540291, 18540295,19540252 ) of the MEXT of Japan. 
[1] H. A. Bethe, Rev. Mod. Phys. 62, 801 (1990).

[2] W. C. Haxton, Phys. Rev. Lett. 60, 1999 (1988).

[3] S. W. Bruenn and W. C. Haxton, Astrophys. J. 376, 678 (1991).

[4] A. Burrows, L. Dessart, C. D. Ott, and E. Livne, Phys. Rep. 442, 23 (2007).

[5] H.-T. Janka, K. Langanke, A. Marek, G. MartinezPinedo, and B. Muller, Phys. Rep. 442, 38 (2007).

[6] N. Ohnishi, K. Kotake, and S. Yamada, Astrophys. J. 667, 375 (2007).

[7] H. Shen, H. Toki, K. Oyamatsu, and K. Sumiyoshi, Prog. Theor. Phys. 100, 1013 (1998).

[8] J. M. Lattimer and F. D. Swesty, Nucl. Phys. A535, 331 (1991).

[9] G. Röpke, A. Grigo, K. Sumiyoshi, and H. Shen, Particles and Nuclei Letters 2, 275 (2005).

[10] G. Röpke, A. Grigo, K. Sumiyoshi, and H. Shen, in Superdense QCD Matter and Compact Stars, edited by D. Blaschke and A. Sedrakian (Springer, Dordrecht, 2006), p. 75 .

[11] G. Röpke, H. Schulz, and L. Münchow, Nucl. Phys. A379, 536 (1982).

[12] M. Schmidt, G. Röpke, and H. Schulz, Ann. Phys. (N.Y.) 202, 57 (1990).

[13] E. O'Connor, D. Gazit, C. J. Horowitz, A. Schwenk, and N. Barnea, Phys. Rev. C75, 055803 (2007).
[14] S. Typel, Phys. Rev. C71, 064301 (2005).

[15] G. Röpke, M. Schmidt, L. Münchow, and H. Schulz, Nucl. Phys. A399, 587 (1983).

[16] C. J. Horowitz and A. Schwenk, Nucl. Phys. A 776, 55 (2006).

[17] J. Margueron, E. van Dalen, and C. Fuchs, Phys. Rev. C76, 034309 (2007).

[18] H. Stein, A. Schnell, T. Alm, and G. Röpke, Z. Phys. A351, 295 (1995).

[19] G. Röpke, M. Schmidt, and H. Schulz, Nucl. Phys. A424, 594 (1984).

[20] K. Sumiyoshi, S. Yamada, H. Suzuki, H. Shen, S. Chiba, and H. Toki, Astrophys. J. 629, 922 (2005).

[21] G. Audi and A. H. Wapstra, Nucl. Phys. A565, 1 (1993).

[22] D. Blaschke, G. Röpke, H. Schulz, A. D. Sedrakian, and D. N. Voskresensky, Mon. Not. R. Astron. Soc. 273, 596 (1995).

[23] S. Ying, W. C. Haxton, and E. M. Henley, Phys. Rev. D40, 3211 (1989).

[24] S. Nakamura, T. Sato, V. Gudkov, and K. Kubodera, Phys. Rev. C63, 034617 (2001).

[25] S. Nakamura, T. Sato, S. Ando, T.-S. Park, F. Myhrer, V. Gudkov, and K. Kubodera, Nucl. Phys. A707, 561 (2002). 